\title{
The role of Movat pentachrome stain and immunoglobulin G4 immunostaining in the diagnosis of autoimmune pancreatitis
}

\author{
Kim E Chu ${ }^{1}$, Bettina G Papouchado ${ }^{1}$, Zhaoli Lane and Mary P Bronner* \\ Department of Anatomic Pathology, Cleveland Clinic, Cleveland, OH, USA
}

\begin{abstract}
Autoimmune pancreatitis is highly responsive to steroid therapy, but because it mimics pancreatic cancer, it often precipitates unnecessary surgery. Adequate diagnostic tests are needed to permit appropriate medical therapy. Lymphocytic and obliterative phlebitis are reported in the majority of cases, as are elevated IgG4positive plasma cells, indicating their high sensitivity. Their specificities, especially when used in conjunction, however, remain largely unknown. Movat pentachrome vascular and IgG4 immunohistochemical stains were performed on a total of 15 autoimmune pancreatitis cases (11 pancreatic resections and 4 biopsies), 39 usualtype alcoholic or idiopathic chronic pancreatitis cases, 35 pancreatic ductal adenocarcinoma-associated chronic pancreatitis cases, and 29 normal pancreata. Marked and diffuse lymphocytic and obliterative venulitis were detected in all 15 cases of autoimmune pancreatitis on Movat staining ( $100 \%$ sensitivity). Only a single carcinoma-associated chronic pancreatitis case among all of the controls showed diffuse benign venulitis that was nonobliterative ( $99 \%$ specificity). Nine of 13,9 autoimmune pancreatitis cases showed marked IgG4 immunopositivity at $\geqslant 10$ positive plasma cells per $\times \mathbf{4 0 0}$ field (69\% sensitivity). No increased IgG4 plasma cells were found in any of 103 controls (100\% specificity). In combination, all of the autoimmune pancreatitis cases had at least one (13/13) and most had both markers (9/13), whereas none of the controls had both markers. Overall, these combined stains show very promising diagnostic utility and should be considered in combination with clinical and serologic analyses in the evaluation of chronic pancreatitis suspicious for malignancy. Future validating studies on preoperative biopsies with outcome data following steroid therapy will be essential.
\end{abstract}

Modern Pathology (2009) 22, 351-358; doi:10.1038/modpathol.2008.196; published online 9 January 2009

Keywords: autoimmune pancreatitis; Movat pentrachrome; venulitis; immunoglobulin G4

Autoimmune pancreatitis is a recently established entity. Its most frequently recognized presentation is that of a pancreatic head mass closely simulating malignancy and often leading to unnecessary Whipple resection for benign and medically treatable disease. ${ }^{1,2}$ Surgical series from Johns Hopkins report that autoimmune pancreatitis represents $21-34 \%$ of false positive cases of Whipple resections for presumed malignancy., ${ }^{3,4}$ Steroid therapy has been shown to be highly and rapidly effective in the majority of cases, leading to resolution within weeks of pancreatic mass lesions, pancreatic and biliary obstruction, and symptoms of pain and

*Correspondence: Professor MP Bronner, MD, Department of Anatomic Pathology, Cleveland Clinic, L25, 9500 Euclid Avenue, Cleveland, OH 44195, USA.

E-mail: bronnem@ccf.org

${ }^{1}$ These authors contributed equally to this work.

Received 30 June 2008; revised and accepted 6 November 2008 published online 9 January 2009 pancreatic insufficiency. ${ }^{5-9}$ Until recently, preoperative diagnosis and medical therapy were not available options, but emerging clinical and pathologic assays are changing this situation. The obvious therapeutic advantage of accurate diagnosis is critical and indicates the need for highly sensitive and specific markers.

Dense lymphoplasmacytic infiltrates and fibrosis of the pancreatic parenchyma and pancreatic duct damage are generally accepted as characteristic findings of autoimmune pancreatitis. These individual histologic findings, however, are commonly observed in other forms of chronic pancreatitis, rendering the diagnosis of autoimmune pancreatitis, especially by biopsy, challenging. Venous pathology, including lymphocytic phlebitis and obliterative phlebitis, is another pathologic aspect reported in the majority of cases of autoimmune pancreatitis; ${ }^{1,10}$ however, the specificity of these vascular changes for autoimmune pancreatitis remains unknown to our knowledge. Because the smooth muscle layers of 
veins in obliterative phlebitis are destroyed, obliterative phlebitis usually blends into the background diffuse inflammatory pancreatitis when viewed on hematoxylin and eosin (H\&E) staining alone. Movat pentachrome staining markedly enhances vessel wall structure, particularly the elastin and smooth muscle components, and thereby greatly enhances the detection of easily overlooked vascular pathology. Examination of pancreatic parenchyma for paired veins in relation to each pancreatic artery, on both Movat and H\&E stains, is another approach that has not been well addressed in the literature but also markedly enhances detection of venous pathology.

Recent studies have also found that autoimmune pancreatitis is often associated with increased IgG4 serum levels and increased IgG4-positive plasma cells by immunohistochemistry within the inflamed pancreatic tissue. ${ }^{11,12}$ This marker appears to be a sensitive diagnostic aid and as Zhang et $a l^{13}$ have shown, can be used to differentiate autoimmune pancreatitis from other common pancreatic pathologies. Its use, however, in combination with or in comparison to Movat staining is essentially unknown.

We aimed to explore the utility of both IgG4 and Movat staining in the diagnosis of autoimmune pancreatitis and in particular, to examine the specificities of these assays against the common pancreatic disorders in the differential. The pertinent controls for specificity testing of any histologic marker of chronic inflammatory pancreatic disease include pancreatic ductal adenocarcinoma and its associated chronic pancreatitis, benign usual-type chronic pancreatitis of either alcoholic or idiopathic etiologies, and normal pancreas. Study of these three control groups relative to documented cases of autoimmune pancreatitis forms the basis of this report.

\section{Materials and methods}

The Cleveland Clinic Internal Review Board approved this research. The pathology archives were searched between the years of 1985 and 2005 for pancreatic resections or core biopsies with a final diagnosis of chronic pancreatitis of any type. All cases were reviewed by at least three of the study pathologists. The inclusion criteria for a diagnosis of autoimmune pancreatitis were (1) marked parenchymal fibroinflammatory destruction, (2) periductal lymphoplasmacytic or histiocytic infiltration, and (3) lack of other clinical causes of chronic pancreatitis, particularly absence of alcoholism. Criteria for usual-type chronic pancreatitis included a known clinical diagnosis of alcoholism, along with fibroinflammatory destruction of the pancreatic parenchyma and absence of a family history of pancreatic disease or pancreatic cancer. Such cases without a history of alcoholism were classified as idiopathic cases of usual-type chronic pancreatitis. Normal pancreatic control tissues were derived from patients undergoing resection for benign pancreatic pathology, pancreatic islet cell tumors, or resection of adjacent organs for nonpancreatic malignancies. Clinical data were obtained from each patient's medical record.

\section{Movat Staining}

For Movat staining of venous lesions, whole tissue sections from a total of 15 cases of autoimmune pancreatitis (11 resections, 4 needle biopsies) and 76 controls (all resections) were available. The control cases included 25 usual-type alcoholic or idiopathic chronic pancreatitis, 31 pancreatic ductal adenocarcinoma-associated chronic pancreatitis cases, and 20 normal pancreata. All materials were routinely fixed in either formalin or Hollandes, processed, embedded in paraffin, and sectioned at 5 and $4 \mu \mathrm{m}$ thicknesses for H\&E versus Movat pentachrome staining, respectively, as previously described. ${ }^{14}$

Lymphocytic and/or obliterative venulitis was defined as venous infiltration by lymphocytes with elastin fiber destruction and incorporation of connective tissue fibers and ground substance into the vein wall with or without obliteration of the lumen. The extent of venous pathology was evaluated semiquantitatively as being absent, patchy (if sections contained venous pathology confined to one or a few discrete veins), or diffuse involvement (if sections contained venulitis involving virtually all veins). All pancreatic parenchymal arteries were specifically identified to examine their respective matched veins on each whole tissue section.

\section{IgG4 Immunohistochemistry}

IgG4 immunostaining utilized whole sections of 13 cases of autoimmune pancreatitis, 11 cases of usualtype chronic pancreatitis, and 10 ductal adenocarcinoma-associated chronic pancreatitis cases. Of the 13 autoimmune pancreatitis cases, 3 were needle biopsies, whereas 10 were resections. All of the 11 usual-type and 10 ductal adenocarcinoma-associated chronic pancreatitis cases were resections. Two pancreatic tissue microarrays containing additional cases of 28 usual-type chronic pancreatitis, 25 ductal adenocarcinoma-associated chronic pancreatitis, and 29 normal pancreata were also analyzed. All tissues were routinely fixed in either formalin or Hollandes, processed, embedded in paraffin, and sectioned at $5 \mu \mathrm{m}$. The microarrays were constructed from $1 \mathrm{~mm}$ diameter cores of histologically verified target tissues that were punched and reembedded in triplicate using a standard microarray instrument (Beecher Instruments, Silver Spring, MD, USA). Primary mouse antihuman monoclonal antibody for IgG4 (clone HP6025; Invitrogen, Carlsbad, CA, USA) at a 1:500 dilution was applied to deparaffi- 
nized and rehydrated tissue sections for $60 \mathrm{~min}$ at $37^{\circ} \mathrm{C}$ followed by a diaminobenzidine basic detection kit (Ventana Medical Systems, Tucson, AZ, USA) and hematoxylin counterstaining on a Benchmark XT automated immunohistochemical stainer (Ventana Medical Systems).

Positive IgG4 plasma cells were scored semiquantitatively based upon the number of positive cells per $\times 400$ high power field (Olympus B42 F/X microscope with a $\times 400$ field area of $0.189 \mathrm{~mm}^{2}$; Olympus, Tokyo, Japan), as follows: $0=$ no staining, $1+=<10$ positive cells, $2+=10-30$ positive cells, and $3+=>30$ positive cells. All available microscopic fields were systematically scored on each tissue section to determine the distribution of staining as either absent, patchy (positive cells found in $<90 \%$ of total microscopic fields), or diffuse (positive cells found in $\geqslant 90 \%$ of total microscopic fields).

\section{Results}

\section{Clinical Features}

The autoimmune pancreatitis patients were comprised of seven women and eight men. The mean age was 56.5 years (range $24-78$ years). Abdominal pain, weight loss, and jaundice were the predominant presenting symptoms. None of the patients had a history of alcohol abuse, family history of pancreatitis, or other predisposing factors for chronic pancreatitis. Computed tomography scans were available in twelve patients, revealing a solid mass in eight, diffuse pancreatic enlargement in three, and no radiologic alterations in one case. On endoscopic retrograde pancreatography performed in eleven cases, the main pancreatic duct was dilated in six cases, narrowed in four cases, and normal in one case. The preoperative diagnosis was pancreatic carcinoma in twelve cases, chronic pancreatitis in two cases, and chronic pancreatitis versus pancreatic carcinoma in one case. Whipple resections were performed in six patients and distal pancreatectomies in five. Four patients underwent needle biopsy alone and avoided surgery.

The chronic pancreatitis and normal pancreata controls were comprised equal numbers of men and women with a mean age of 60 years (range 36-82 years). In the chronic pancreatitis cases, abdominal pain, and weight loss were again the predominant presenting symptoms. Imaging studies in $85 \%$ of the patients showed a mass or cystic lesion in the pancreas. Surgical treatment consisted of Whipple resection in $65 \%$ of cases and distal pancreatectomy in the remaining $35 \%$. The normal pancreata sections were derived from pancreatic resections for islet cell tumors ( $52 \%)$, benign cystic lesions $(14 \%)$, benign pancreatic ductal stricture (3\%), and in conjunction with resections of malignancies in other organs $(31 \%)$.

\section{Movat Staining}

Marked lymphocytic venulitis with elastin fiber destruction and incorporation of connective tissue fibers and ground substance into the vein wall was detected in all 15 cases of autoimmune pancreatitis (Figure 1a and b; Table 1). Moreover, the venous pathology was diffuse in distribution in all of the autoimmune pancreatitis cases, involving virtually all of the veins and was easily detected in both pancreatic needle biopsies $(n=4)$ and resection specimens $(n=11)$. Five cases further showed obliterative venous changes. In contrast, none of the usual-type chronic pancreatitis cases and only a single ductal adenocarcinoma-associated chronic pancreatitis case showed diffuse venous inflammatory involvement. This single cancer-related case also lacked venous invasion by pancreatic ductal adenocarcinoma. Venulitis in conjunction with venous invasion by pancreatic ductal adenocarcinoma was excluded from this analysis, as it does not pose a diagnostic dilemma in the differential of pancreatic cancer versus possible autoimmune pancreatitis. Of 76, 65 controls had no detectable venous pathology (Figure 1c and d), whereas patchy venous changes were observed in the remaining five usual-type and five ductal adenocarcinoma-associated chronic pancreatitis cases (Figure 1e and f). Diffuse, marked lymphocytic venulitis/obliteration by Movat staining was found to be $100 \%$ sensitive and $99 \%$ specific for autoimmune pancreatitis. Comparatively, Movat staining was still $86 \%$ specific when allowing for any degree of venous pathology.

\section{IgG4 Immunostaining}

The IgG4 immunostain demonstrated uniformly intense staining of plasma cell cytoplasm and showed virtually no artifactual background staining of other cell types. As seen in Table 1, the IgG4 immunostain is highly specific $(100 \%)$, staining none of the pertinent controls of ductal adenocarcinoma-associated or usual-type chronic pancreatitis or normal pancreatic tissue. Its sensitivity in our hands, however, was somewhat less than reported in literature series. ${ }^{11}$ Using a $2+$ or $3+$ staining cutoff ( $>10$ plasma cells per $\times 400$ field), $69 \%$ of clinicopathologically verified cases of autoimmune pancreatitis were positive. All five of the $3+$ stained cases were diffusely stained (approximately 10 positive plasma cell per 10 high power fields; mean $=222$ positive cells in 228 fields counted). All of the $2+$ stained cases showed patchy staining (approximately 6 positive plasma cells per 10 high power fields; mean $=220$ positive plasma cells in 400 fields counted), and the $1+$ stained case was patchy (approximately 4 positive plasma cells per 10 high power fields; 60 positive plasma cells in 140 fields counted). Two of the $3+$ cases were needle 

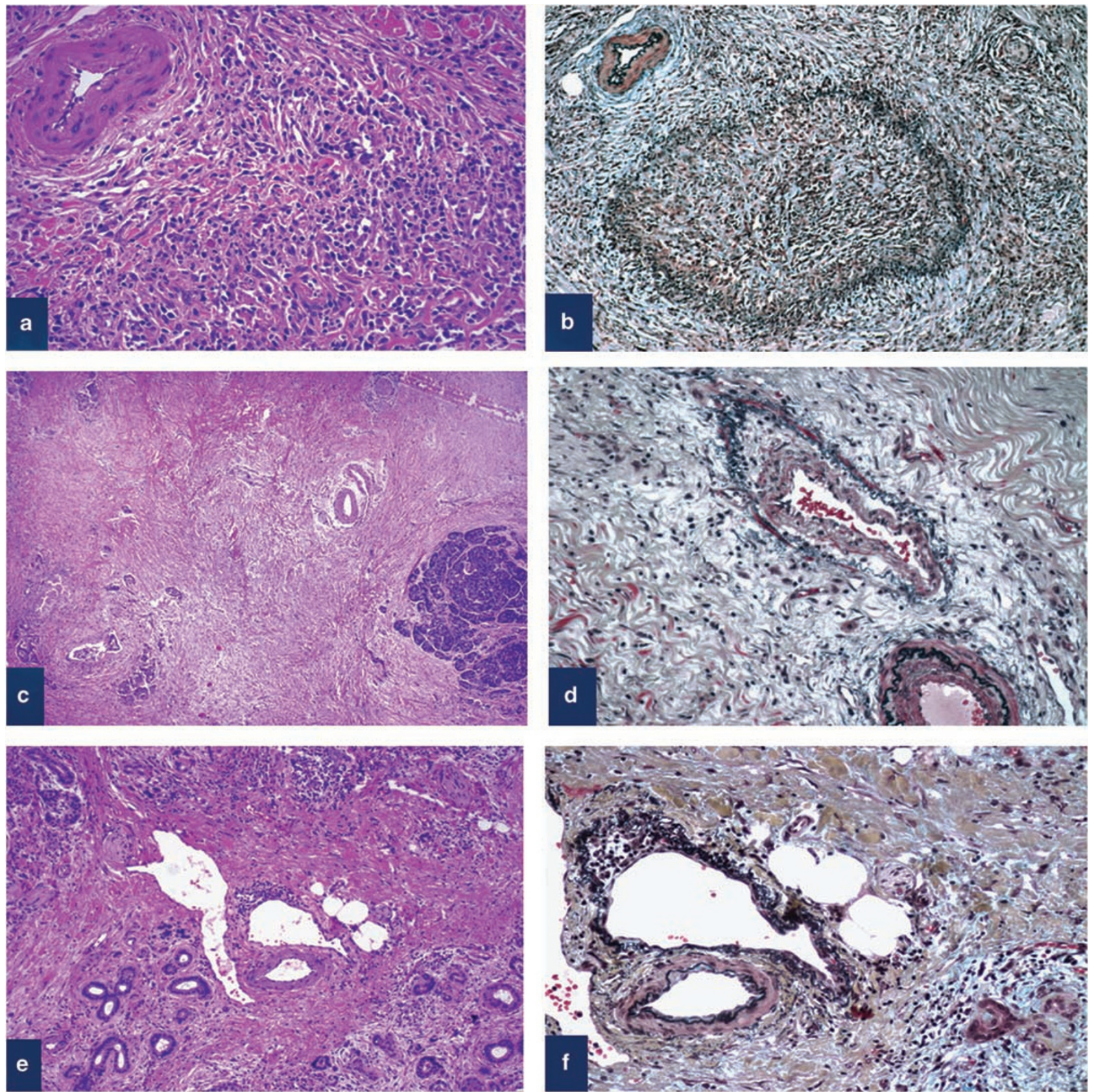

Figure 1 (a) Autoimmune pancreatitis involving and replacing pancreatic lobules by marked interstitial fibrosis and lymphoplasmacytic infiltration. Note the apparent unpaired artery without an obvious accompanying vein (H\&E). (b) Movat pentachrome stain on the same vessels shown on H\&E in panel a, illustrating the Movat's dramatic enhancement of obliterative venous pathology in autoimmune pancreatitis. Fibrosis and lymphoplasmacytic infiltration destroy the vein wall and disrupt elastin fibers, resulting in luminal occlusion (Movat pentachrome). (c) Usual-type chronic pancreatitis caused by alcoholism (H\&E). (d) Alcoholic pancreatitis demonstrating a normal vein and paired artery with intact elastica, intima, and media (Movat pentachrome). (e) Pancreatic ductal adenocarcinoma-associated chronic pancreatitis (adenocarcinoma absent from this field; H\&E). (f) Pancreatic ductal adenocarcinoma-associated chronic pancreatitis showing mild and patchy lymphocytic phlebitis with mild elastin fiber irregularity (Movat pentachrome).

biopsies. The remaining single needle biopsy case showed no IgG4 immunostaining.

\section{Combined Testing}

The combined results on a per case basis for both venulitis on Movat staining and IgG4 immunohistochemical staining of plasma cells are shown in Table 2.

\section{Discussion}

Autoimmune. pancreatitis is a benign fibroinflammatory variant of chronic pancreatitis that typically presents with pain, weight loss, obstructive jaundice, and a diffuse or localized pancreatic mass mimicking cancer. It often leads to unnecessary surgical resection, ${ }^{1-4}$ which is particularly tragic 
Table 1 Movat and IgG4 staining results in autoimmune pancreatitis and controls

\begin{tabular}{|c|c|c|c|c|}
\hline & $\begin{array}{l}\text { Autoimmune } \\
\text { pancreatitis }\end{array}$ & $\begin{array}{l}\text { Usual-type chronic } \\
\text { pancreatitis }\end{array}$ & $\begin{array}{c}\text { Pancreatic ductal } \\
\text { adenocarcinoma-associated chronic } \\
\text { pancreatitis }\end{array}$ & $\begin{array}{l}\text { Normal } \\
\text { pancreas }\end{array}$ \\
\hline \multicolumn{5}{|c|}{ Movat staining venulitis } \\
\hline Diffuse & $100 \%(15 / 15)$ & $0 \%(0 / 25)$ & $3.2 \%(1 / 31)$ & $0 \%(0 / 20)$ \\
\hline Patchy & $0 \%(0 / 15)$ & $20 \%(5 / 25)$ & $16 \%(5 / 31)$ & $0 \%(0 / 20)$ \\
\hline Obliterative & $33 \%(5 / 15)$ & $0 \%(0 / 25)$ & $0 \%(0 / 31)$ & $0 \%(0 / 20)$ \\
\hline \multirow[t]{4}{*}{ IgG4 staining } & $3+(5 / 13)$ & & & \\
\hline & $2+(4 / 13)$ & & & \\
\hline & $1+(1 / 13)$ & & & \\
\hline & $0(3 / 13)$ & $0(39 / 39)$ & $0(35 / 35)$ & $0(29 / 29)$ \\
\hline
\end{tabular}

Table 2 Synchronous Movat and IgG4 staining results

\begin{tabular}{|c|c|c|c|c|}
\hline \multirow{3}{*}{$\begin{array}{l}\text { IgG4-positive } \\
(\geqslant 10 \text { plasma cells per } \times 400 \text { field })\end{array}$} & \multicolumn{2}{|c|}{$\begin{array}{c}\text { Movat-positive } \\
\text { (diffuse } \pm \text { obliterative venulitis) }\end{array}$} & \multicolumn{2}{|c|}{$\begin{array}{c}\text { Movat-negative } \\
\text { (minimal or absent venulitis) }\end{array}$} \\
\hline & AIP & $9 / 13$ & AIP & $0 / 13$ \\
\hline & Usual-type CP & $0 / 25$ & Usual-type CP & $0 / 25$ \\
\hline & CA-assoc CP & $0 / 31$ & CA-assoc CP & $0 / 31$ \\
\hline & Normal & $0 / 20$ & Normal & $0 / 20$ \\
\hline \multirow{4}{*}{ IgG4-negative $(<10$ plasma cells per $\times 400$ field $)$} & AIP & $4 / 13$ & AIP & $0 / 13$ \\
\hline & Usual-type CP & $0 / 25$ & Usual-type CP & $25 / 25$ \\
\hline & CA-assoc CP & $1 / 31$ & CA-assoc CP & $30 / 31$ \\
\hline & Normal & $0 / 20$ & Normal & $20 / 20$ \\
\hline
\end{tabular}

AIP, autoimmune pancreatitis; CP, chronic pancreatitis; CA, carcinoma; assoc, associated.

given that this entity is now known to be rapidly and effectively treated by corticosteroid therapy. ${ }^{5-9}$ Accordingly, preoperative biopsy diagnosis and medical treatment are highly sought after clinical goals. This study sheds further light on the issue by demonstrating the utility of two histologic features in combination, namely venous pathology as detected by Movat staining, in conjunction with increased pancreatic tissue IgG4-positive plasma cells by immunohistochemistry. Although the high sensitivity for both tests has been established in the literature, their near perfect specificity, particularly in combination, remained largely unknown before this study.

Some authors contend that neither preoperative biopsy nor intraoperative frozen sections are indicated for pancreatic lesions suspicious for cancer, as surgical resection will be required regardless of the findings. ${ }^{15-17}$ This approach conflicts with evolving knowledge that pancreatic cancer is rarely curable by surgery, ${ }^{18}$ and has been based upon the following: (1) advances in imaging have improved radiologic pancreatic cancer diagnosis, (2) pancreaticoduodenectomy (Whipple resection) can have low mortality in high volume centers, (3) decisions to operate are more often dictated by clinical findings than histopathology, (4) unsampled cancer can never be excluded by focal biopsies, and (5) good medical therapy was previously lacking for benign masses/ obstruction. ${ }^{19-21}$ This approach must now be reevaluated in light of autoimmune pancreatitis and its mimicry of cancer, its increasingly specific diagnostic tests, and its dramatic response to steroid therapy. As the most common cause of unnecessary Whipple resection for benign disease, ${ }^{3}$ accurate preoperative diagnosis of autoimmune pancreatitis is important.

An accepted histologic feature of autoimmune pancreatitis is lymphocytic venulitis, as included in the Mayo Clinic diagnostic criteria, ${ }^{22}$ whereby lymphocytes surround and infiltrate the walls of medium to large-sized pancreatic veins and may lead to occlusive obliteration or destruction of the vessels (Figure 1a and b). Movat staining amplifies the detection of venulitis and obliterative change in autoimmune pancreatitis many fold over H\&E staining alone. Identification of the pancreatic arteries, which are noticeably unaffected in autoimmune pancreatitis but characteristically paired with veins, further improves the detection of inconspicuous and damaged veins in this disease.

Specificity data for venulitis in autoimmune pancreatitis are not well established. The striking intensity and diffuseness of the venulitis in autoimmune pancreatitis led us to speculate that this histologic feature may be more specific, as our reported data now confirm. The pertinent specificity control groups, including usual-type chronic pancreatitis of either alcoholic or idiopathic causes $(n=25)$, pancreatic ductal adenocarcinoma $(n=31)$, 
and normal pancreata $(n=20)$, lacked diffuse venous pathology, in all but a single case of cancerassociated chronic pancreatitis that did have diffuse lymphocytic but nonobliterative venulitis. Thus, our data demonstrate that diffuse and/or obliterative lymphocytic venulitis, as highlighted by Movat staining, is not only $100 \%$ sensitive for, but also $99 \%$ specific for autoimmune pancreatitis.

Of note, patchy and mild venulitis was observed in $16 \%$ ( 5 of 31 ) of chronic pancreatitis cases associated with pancreatic cancer and $20 \%$ (5 of 25) of usual-type chronic pancreatitis cases of alcoholic or idiopathic types in our study. From these data, the finding of any degree of venulitis is somewhat less specific at $86 \%$ relative to diffuse and/or obliterative venulitis at 99\% specificity. However, when no venous change is found on Movat staining, it appears to be highly unlikely that the patient has autoimmune pancreatitis (the negative predictive value in our study population is 65 of 65 cases or $100 \%$; Table 1).

On H\&E staining alone, venulitis can be very difficult to appreciate, such as at the time of frozen section when additional vascular stains are not available. Its identification, however, even on $\mathrm{H} \& \mathrm{E}$ alone, can be greatly enhanced by first identifying the pancreatic arteries and then looking for the characteristically paired veins. The arteries are far easier to find on screening H\&E microscopy, based upon their thicker muscular walls, more prominent elastica, and more open lumens. Once the arteries are identified, it becomes far more obvious whether the accompanying vein is damaged or not visible at all.

Immunohistochemical staining for IgG4 has also shown promise in the workup of preoperative pancreatic masses without histologic evidence of tumor on biopsy. IgG4 comprises approximately $4 \%$ of the total circulating serum IgG in normal adults, but has been reported to be elevated in autoimmune pancreatitis in $62-94 \%$ of patients. ${ }^{23-28}$ Increasingly, studies have also found elevated numbers of IgG4positive plasma cells by tissue immunohistochemistry in autoimmune pancreatitis. Most recently, Deheragoda et al ${ }^{11}$ demonstrated an $85 \%$ sensitivity in autoimmune pancreatitis patients for >10 IgG4positive plasma cells per high power field in both pancreatic and extrapancreatic tissues in comparison to $0.6 \%$ of specimens from controls of normal pancreata $(n=10)$ and pancreatic ductal adenocarcinomas $(n=20)$. Increased tissue IgG4-positive plasma cells were found irrespective of the patients' serum IgG4 levels, indicating the particular usefulness of the stain in autoimmune pancreatitis patients with normal serum IgG4 levels. Klöppel and colleagues found that in periductal areas $>20$ IgG4-positive plasma cells per high power field using a $\times 40$ lens had high specificity $(100 \%)$, but only moderate sensitivity ( $43 \%$ ) in their series of 51 autoimmune pancreatitis resections in comparison to nonautoimmune chronic pancreatitis cases
( $n=19$ controls). ${ }^{29}$ Our findings corroborate the high specificity $(100 \%)$ of IgG4 staining in 103 additional controls. Its sensitivity, however, was similarly less in our study, with only $69 \%$ of the clinicopathologically verified autoimmune pancreatitis cases having increased IgG4-positive plasma cells ( $>10$ plasma cells per $\times 400$ field). Part of the sensitivity discrepancy may relate to the fact that three $(23 \%)$ of our autoimmune pancreatitis cases had only needle core biopsies and therefore less tissue for examination. The amount of tissue examined is pertinent to the analysis of focal or patchy findings because of the potential for sampling error. Most of our positive autoimmune pancreatitis cases showed diffuse plasma cell staining $(n=5$, all $3+)$, but some of the positive cases were patchy $(n=4$, all $2+$ cases). Thus, although a positive IgG4 result appears to be highly informative, a negative result is not based on its imperfect sensitivity.

A current area of interest in autoimmune pancreatitis not surprisingly relates to preoperative needle core biopsies to preempt surgery and permit a trial of steroid therapy. The diagnosis of autoimmune pancreatitis by needle biopsy is challenging due both to the patchiness of IgG4-positive plasma cells and the limited nature of biopsy sampling. As expected, the literature provides mixed results on the utility of biopsy diagnosis. Zhang et $a l^{13}$ found promising results with all nine of the biopsies in their study showing at least mild numbers of IgG4-positive cells. Chari et a ${ }^{22}$ also evaluated 16 core biopsies and although only $44 \%$ (7 of 16) had the full histologic characteristics of autoimmune pancreatitis, 94\% (15 of 16) had abundant IgG4-positive plasma cells. However, Bang et $a l^{30}$ observed abundant IgG4-positive cells ( $>10$ cells per high power field) in only $21 \%$ (4 of 19) of ultrasound-guided core biopsies. In our limited evaluation of this issue, only $66 \%$ (2 of 3 ) of the autoimmune pancreatitis biopsies were positive for IgG4, although both did show $3+$ positivity. Unfortunately, the imperfect sensitivity of $\operatorname{IgG4}$ staining for autoimmune pancreatitis further highlights the problem of relying too heavily on this one feature in making the diagnosis. Bang et $a l^{30}$ also found abundant IgG4-positive cells in the pancreas in two of eight patients with chronic alcoholic pancreatitis and one of ten patients with pancreatic cancer, raising concern about specificity, in contrast to our and others ${ }^{11,29}$ much higher specificity data. Inflammatory myofibroblastic tumors and sclerosing mesenteritis lesions involving the pancreas have also been shown to be associated with positive IgG4 plasma cells. ${ }^{31}$

These confounding factors for IgG4 alone led us to consider a combination of Movat and IgG4 staining in conjunction with morphology in the diagnosis of autoimmune pancreatitis, an approach that we have shown to be helpful. In fact, all of our false negative IgG4 cases in autoimmune pancreatitis, including 
both resections and biopsies, had the diffuse lymphocytic venulitis permitting the diagnosis despite negative IgG4 results. In turn, the single false positive diffuse venulitis case in a cancer control was negative for IgG4. None of the 103 controls showed dual positivity. These results show that autoimmune pancreatitis is virtually certain in the presence of one of the two markers and definitive in the presence of both. It should be noted, however, that rare cases of coexistent pancreatic ductal adenocarcinoma and autoimmune pancreatitis have been reported. ${ }^{32}$ These highlight the need for continued close evaluation of autoimmune pancreatitis patients for undiagnosed cancer and to ensure resolution of their signs and symptoms following steroid therapy. ${ }^{32}$

In summary, the diagnosis of autoimmune pancreatitis is challenging and requires complete correlation of all available histologic, serologic, radiographic, and clinical data. In combination, Movat vascular and IgG4 immunohistochemical plasma cell staining are now shown to be an additional helpful histologic approach. Finally, it should again be emphasized that this study was performed almost entirely on pancreatic resection specimens and may not therefore be fully comparable to needle biopsy specimens in preoperative settings. Future outcome studies on preoperative needle biopsies combined with therapeutic steroid trials will be essential confirmatory steps in assessing the diagnostic utility of these tests.

\section{Disclosure/conflict of interest}

The authors state no conflict of interest.

\section{References}

1 Toomey DP, Swan N, Torreggiani W, et al. Autoimmune pancreatitis. Br J Surg 2007;94:1067-1074.

2 Krasinskas AM, Raina A, Khalid A, et al. Autoimmune pancreatitis. Gastroenterol Clin North Am 2007;36:239-257.

3 Abraham SC, Wilentz RE, Yeo CJ, et al. Pancreaticoduodenectomy (Whipple resections) in patients without malignancy: are they all 'chronic pancreatitis'? Am J Surg Pathol 2003;27:110-120.

4 Hardacre JM, Iacobuzio-Donahue CA, Sohn TA, et al. Results of pancreaticoduodenectomy for lymphoplasmacytic sclerosing pancreatitis. Ann Surg 2003;237:853-858.

5 Nishino T, Toki $\mathrm{F}$, Oyama $\mathrm{H}$, et al. Biliary tract involvement in autoimmune pancreatitis. Pancreas 2005;30:76-82.

6 Hamano H, Kawa S, Horiuchi A, et al. High serum IgG4 concentrations in patients with sclerosing pancreatitis. N Engl J Med 2001;344:732-738.

7 Kamisawa T, Yoshiike M, Egawa N, et al. Treating patients with autoimmune pancreatitis: results from a long-term follow-up study. Pancreatology 2005;5: 234-238.
8 Takayama M, Hamano H, Ochi Y, et al. Recurrent attacks of autoimmune pancreatitis result in pancreatic stone formation. Am J Gastroenterol 2004;99: 932-937.

9 Tanaka S, Kobayashi T, Nakanishi K, et al. Corticosteroid-responsive diabetes mellitus associated with autoimmune pancreatitis. Lancet 2000;356:910-911.

10 Klöppel G, Luttges J, Sipos B, et al. Autoimmune pancreatitis: pathological findings. JOP 2005;6:97-101.

11 Deheragoda MG, Church NI, Rodriguez-Justo M, et al. The use of immunoglobulin G4 immunostaining in diagnosing pancreatic and extrapancreatic involvement in autoimmune pancreatitis. Clin Gastroenterol Hepatol 2007;5:1229-1234.

12 Deshpande V, Chicano S, Finkelberg D, et al. Autoimmune pancreatitis: a systemic immune complex mediated disease. Am J Surg Pathol 2006;30: 1537-1545.

13 Zhang L, Notohara K, Levy MJ, et al. IgG4-positive plasma cell infiltration in the diagnosis of autoimmune pancreatitis. Mod Pathol 2007;20:23-28.

14 Prophet EB, Mills B, Arrington JB, et al. Armed Forces Institute of Pathology Laboratory Methods in Histotechnology. American Registry of Pathology: Washington, DC, 1992.

15 Warshaw AL. Implications of peritoneal cytology for staging of early pancreatic cancer. Am J Surg 1991;161:26-29.

16 Makary MA, Warshaw AL, Centeno BA, et al. Implications of peritoneal cytology for pancreatic cancer management. Arch Surg 1998;133:361-365.

17 Levy MJ, Wiersema MJ, Chari ST. Chronic pancreatitis: focal pancreatitis or cancer? Is there a role for FNA/biopsy? Autoimmun Pancreatitis. Endosc 2006;38:S30-S35.

18 Ducreux M, Boige V, Goéré D, et al. The multidisciplinary management of gastrointestinal cancer. Pancreatic cancer: from pathogenesis to cure. Best Pract Res Clin Gastroenterol 2007;21:997-1014.

19 Clarke D, Clarke B, Thomson S, et al. The role of preoperative biopsy in pancreatic cancer. HPB (Oxford) 2004;6:144-153.

20 Brennan MF. Surgical treatment of periampullary and pancreatic cancer. Eur Surg 2002;34:25-26.

21 Taylor B. Carcinoma of the head of the pancreas versus chronic pancreatitis: diagnostic dilemma with significant consequences. World J Surg 2003;27:1249-1257.

22 Chari ST, Smyrk TC, Levy MJ, et al. Diagnosis of autoimmune pancreatitis: the Mayo Clinic experience. Clin Gastroenterol Hepatol 2006;4:1010-1016.

23 Morell A, Skvaril F, van Loghem E, et al. Human IgG subclasses in maternal and fetal serum. Vox Sang 1971;21:481-492.

24 Kamisawa T, Funata N, Hayashi Y, et al. A new clinicopathological entity of IgG4-related autoimmune disease. J Gastroenterol 2003;38:982-984.

25 Okazaki K, Uchida K, Chiba T. Recent concept of autoimmune-related pancreatitis. J Gastroenterol 2001;36:293-302.

26 Kamisawa T, Okamoto A, Funata N. Clinicopathological features of autoimmune pancreatitis in relation to elevation of serum IgG4. Pancreas 2005;31:28-31.

27 Hirano K, Shiratori Y, Komatsu Y, et al. Involvement of the biliary system in autoimmune pancreatitis: a follow-up study. Clin Gastroenterol Hepatol 2003;1:453-464. 
28 Hirano K, Kawabe T, Yamamoto N, et al. Serum IgG4 concentrations in pancreatic and biliary diseases. Clin Chim Acta 2006;367:181-184.

29 Klöppel G, Sipos B, Zamboni G, et al. Autoimmune pancreatitis: histo- and immunopathological features. J Gastroenterol 2007;42:28-31.

30 Bang SJ, Kim MH, Kim do H, et al. Is pancreatic core biopsy sufficient to diagnose autoimmune chronic pancreatitis? Pancreas 2008;36:84-89.
31 Uchida K, Sohei S, Hideaki M, et al. Inflammatory pseudotumors of the pancreas and liver with infiltration of IgG4-positive plasma cells. Int Med 2007;46:1409-1412.

32 Witkiewicz AK, Kennedy EP, Kennyon L, et al. Synchronous autoimmune pancreatitis and infiltrating pancreatic ductal adenocarcinoma: case report and review of the literature. Hum Pathol 2008;39: 1548-1551. 